\title{
A Hybrid Approach to Brain Extraction from Premature Infant MRI
}

\author{
Michèle Péporté ${ }^{1}$, Dana E. Ilea Ghita ${ }^{1}$, Eilish Twomey ${ }^{2}$, and Paul F. Whelan ${ }^{1}$ \\ 1 Centre for Image Processing and Analysis, Dublin City University, Ireland \\ 2 Department of Radiology, Childrens University Hospital, Dublin, Ireland \\ michele.peporte2@mail.dcu.ie, danailea@eeng.dcu.ie, \\ eilish.twomey@cuh.ie, paul.whelan@dcu.ie
}

\begin{abstract}
This paper describes a novel automatic skull-stripping method for premature infant data. A skull-stripping approach involves the removal of non-brain tissue from medical brain images. The new method reduces the image artefacts, generates binary masks and multiple thresholds, and extracts the region of interest. To define the outer boundary of the brain tissue, a binary mask is generated using morphological operators, followed by region growing and edge detection. For a better accuracy, a threshold for each slice in the volume is calculated using k-means clustering. The segmentation of the brain tissue is achieved by applying a region growing and finalized with a local edge refinement. This technique has been tested and compared to manually segmented data and to four well-established state of the art brain extraction methods.
\end{abstract}

Keywords: Skull Stripping, Newborns MRI, Brain Segmentation.

\section{Introduction}

In this study, we focus on Magnetic Resonance Imaging (MRI) brain segmentation from premature infants. Premature birth is associated with a high risk of an injury in white matter. This brain injury can cause the development of cerebral palsy [12] 26]. Therefore, the segmentation of newborn brain MRI is an important task for the study and diagnosis of neurodevelopment disorders at an early stage. The first stage of brain segmentation involves the extraction of the entire region of interest (ROI) which consists of the brain tissue such as cortical grey matter (GM), white matter (WM), deep grey matter, and cerebellum. This procedure is called skull-stripping and requires removing the skull, fat and cerebrospinal fluid (CSF) parts. Skull-stripping is a difficult task on adult brain MRI. However, it is more challenging using premature infant brain MRI because infants are still in an early development stage of the brain structure. Harnsberger et al. 8] provides an useful insight into the development of the newborn brain MRI and the undergoing changes in the brain structure during the first years of age.

Over the past years, various techniques have been proposed for unsupervised skull stripping such as histogram-based [2] 20, region-based [7] 22], boundarybased [23], graph-cut based [18, fuzzy-based [9] or hybrid approaches [4] [19].

A. Heyden and F. Kahl (Eds.): SCIA 2011, LNCS 6688, pp. 719 730, 2011.

(C) Springer-Verlag Berlin Heidelberg 2011 
Some of the methods have been embedded in software tools such as BrainSuite [21, SPM8 [25], MRIcroN [17] or FMRIB Software Library (FSL) [5] 24]. Brain Surface Extraction (BSE) 22 is one of the well-established Brain Extraction Algorithms (BEA). BSE is an edge based method which uses an anisotropic diffusion filter, followed by a Marr and Hildreth edge detector. The final segmentation is obtained by applying morphological operators on the edge map to enable the removal of the non-brain tissue. The second well-established BEA is called Brain Extraction Tool (BET) 23. This method is based on estimating the intensity threshold of the brain and non-brain regions, and then determines the centre of gravity of the brain volume, followed by defining the initial sphere which is based on the previous calculated centre of gravity. Finally, the technique deforms the initial sphere outwards to the brain tissue boundaries. The third well-known brain segmentation approach is called Statistical Parametric Mapping (SPM) 6] which consists of realigning, normalizing and segmenting steps. Realigning and normalizing were performed to transform the volume into the Talairach space. The segmentation generates GM, WM and CSF areas.

Only a few methods have been developed with the main focus on brain extraction from MRI data from infants. One of these approaches was proposed by Chiverton et al. 4. Their technique first removes the background using region growing, then uses parameter estimation to fit an intensity Gaussian mixture model to a predefined histogram. A 2D mask is created by segmentation using thresholding and region growing. The final segmentation is achieved using simple 3D morphological operators. Another approach based on infant brain MRI was proposed by Kobashi et al. 9. This technique uses fuzzy rule-based active surface models. The images were segmented using thresholding and morphological operations. A surface model was achieved using connected triangles which allow the surface to be deformed by moving them around. The positions of the triangles were defined using fuzzy IF-THEN rules.

The aim of this paper is to present a novel skull-stripping method called Hybrid Skull-Stripping (HSS) which removes all non-brain tissue in brain MRIs using premature infant data. At this stage, the region of interest is composed of cortical grey matter, deep grey matter, white matter and the cerebellum. The removal parts are skull, fat, fluid, eyes and body parts. According to our knowledge, BET and BSE have been used by the majority of the previous developed skull-stripping approaches for comparison purposes. In this paper, we use BET, BSE and SPM to compare with the new method.

\section{Proposed Method}

\subsection{Overview}

Figure 1 presents an overview on the structure of the HSS algorithm proposed in this paper. The pre-processing step deals with the improvement of the image quality. This step is divided in two parts, first the image is smoothed to reduce noise and the second part implicates intensity adjustment to remove the intensity shifts between slices throughout the volume. The next step comprises the 


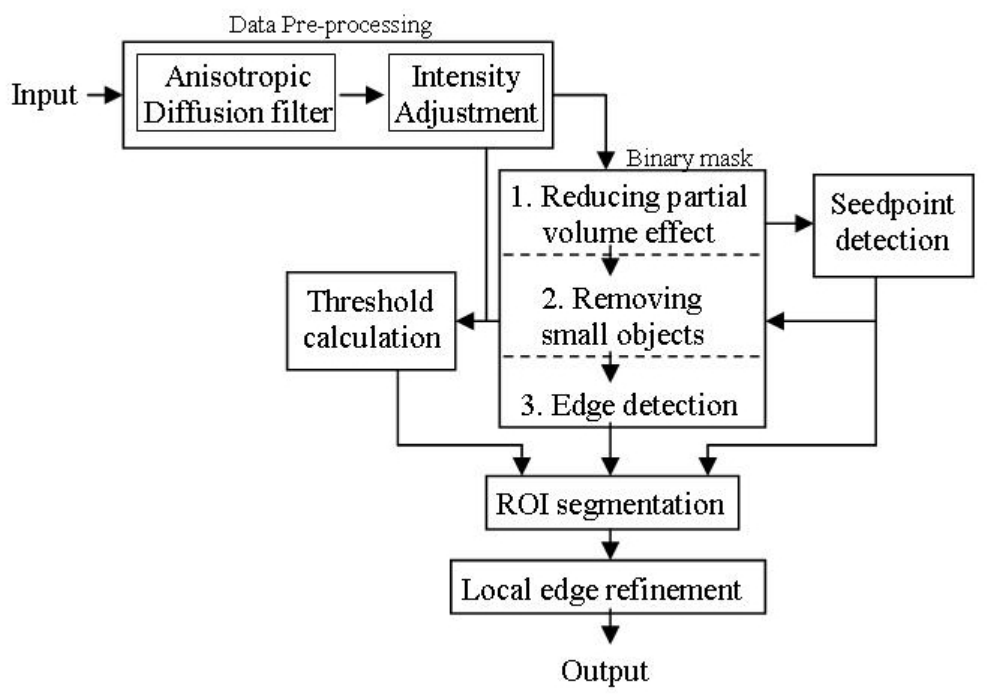

Fig. 1. Overview of the proposed skull stripping technique

generation of a binary mask. The mask is created within three steps, first reducing the partial volume effect, second removing small objects and finally detecting the outer boundaries of the brain tissues. Before the ROI can be segmented, a seed point is automatically defined and a threshold for each image in the volume is calculated. The final step refines the outer edges.

\subsection{Data Pre-processing}

One challenge in medical images is generated by the inconsitencies appearing in the images between patients and throughout the patient volumes. In order to address this issue we propose to apply a two-step procedure. The first step addresses the noise reduction in the images while preserving the edges, while the second step deals with the adjustment of intensity changes in all slices of the dataset.

Anisotropic diffusion filter. The Coherence Enhancing Diffusion Filter (CED) from Weickert 27] allows us to smooth the image and strengthen the edges. In previous skull stripping approaches [22] [28, a similar anisotropic diffusion filter 13. has been used to strengthen the edges between each region. This allows a more precise removal of the non-brain tissue and it facilitates the separation of each region of interest such as WM and GM. This works well on adult MRIs. However, when using infant brain MRIs especially preterm children, this task is more complicated and this is caused by a higher quantity of Partial Volume 
Effect (PVE). In our case, the focus of applying of the anisotropic diffusion filter lies in strengthening the edges between the brain tissue and the CSF in order to facilitate the application of an edge detector in a later stage of the algorithm. Experimentally, we concluded that the best results were obtained when setting the CED paramenters to the following values: $\sigma=0.5, \rho=4$.

Intensity Adjustment. Due to the MRI acquisition procedure, MRIs images include intensity changes, not only between patients but also within the same data sequence. The aim of this step is to adjust the ROI intensity into the same range throughout the entire sequence of one patient. In the first part, the background is removed by using a simple tresholding procedure that sets all background pixels to 0 . In the second part, the intensity of the foreground region is adjusted in each image individually. The approximate ROI which includes all brain tissue is detected using histogram analysis. In each histogram, one local maximum and two local minima of the ROI are detected. By knowing the location of the ROI in the histogram, the region is shifted into the same intensity range for each image. To avoid a cut-off in the bright intensity, that area will be stretched out, so a smooth transition is still maintained. Figure 2 presents an example of an image taken before and after the intensity adjustment and its corresponding histogram. The approximate ROI which lies between two local minimums is clearly visible in the histogram. The pixels with intensities smaller than $1 \times 10^{4}$ in the histogram belong to the background. The adjustment of the intensity does not mean that each region has the same intensity throughout the volume but implies that each region can be found in a certain range. The problem which remains to be addressed is that the GM and WM still overlap in their intensity range.
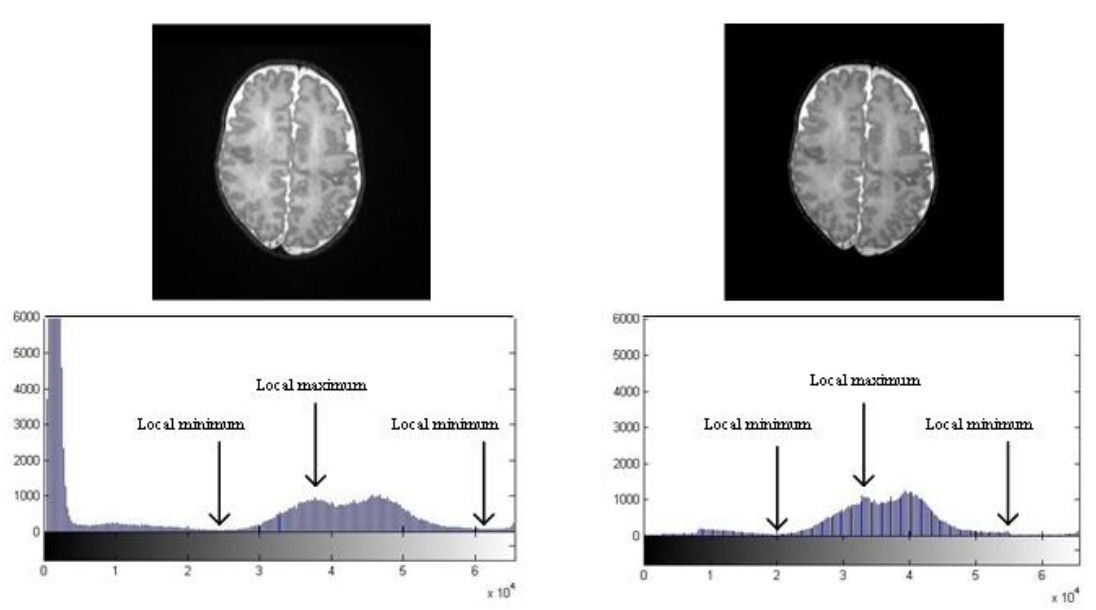

Fig. 2. Left: an sample and its associated histogram before applying the intensity adjustment; right: same sample after the intensity adjument was applied 


\subsection{Creating Mask}

In the first stage, a primary mask of the main brain region is constructed with the intention of reducing the PVE. This is provided by bringing the ROI into the foreground and the other region into the background using once the erosion and dilation operators. In the resulting image, the foreground region will be defined by a different intensity value when compared to the background region.

In the second step, a fast binary region growing is used to check the connectivity of the main brain region. Additionally this enables us to remove regions that are not connected such as the eyes. The automated seedpoint detection for this step is explained in the next sub-section.

To generate the final mask, the Marr and Hildreth edge detector [11 is applied on the second mask that was modified by projecting the intensities on the ROI. The Marr and Hildreth edge detector first runs a Gaussian low-pass filter followed by detecting the boundaries using the Laplacian edge operator. The best results have been obtained using a Gaussian kernel of $5 \times 5$ and a variance $\sigma$ of 2 . The purpose of this final step is to remove the large fluid areas on the outside of the brain region. Due to partial volume effect, in some cases the edge detector does not find enclosed boundaries. Morphological operators have been applied on the edge map to connect loose ends of edges. The images corresponding to each mask generation step are given in Figure 3. In the proposed HSS algorithm, the generation of the binary mask is essential, as it will be used as boundary stopping condition in a later procedure.
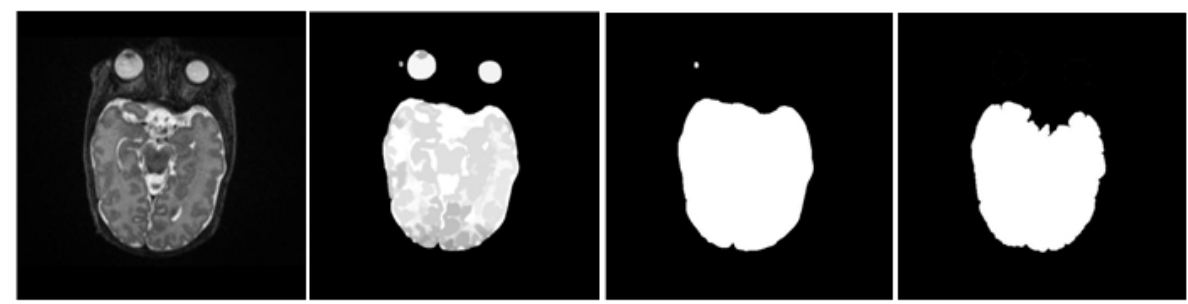

Fig. 3. Displays each mask step, starting with the original image, followed by first mask, then second mask and finish with the final mask. The small bright part visible in the third image is a leftover of the lacrimal glands.

\subsection{Automatic Seedpoint Detection}

The seedpoint is the starting point for the region growing algorithm used in HSS. During the intensity adjustment, the seedpoint in the $\mathrm{z}$ direction is determined by selecting the image with the largest connected region of brain tissue. Within the image of seedpoint $\mathrm{z}$, the $\mathrm{x}$ and $\mathrm{y}$ coordinates are obtained by extracting the largest connected region associated with the dominant intensity. The dominant intensity in ROI is retrieved as a local maximum in the histogram. One pixel will be taken from the extracted region and defined as seedpoint with the coordinate $(\mathrm{x}, \mathrm{y}, \mathrm{z})$. 


\subsection{ROI Segmentation}

To extract the ROI, a region growing algorithm is applied on the MRI sequence. Pohle et al. 14 proposed an adaptive region growing to segment regions in medical images using two runs of the region growing. However, if conditions such as shape differences or intensity changes within the region of interest are not well defined then the method does not work well. Li et al. 10 propose a different region growing method to address this problem. This has been done by using an adaptive threshold based on the mean value and standard deviation of the region of interest to define the grey value range of the current pixel.

We tried different thresholds such as the adaptive threshold proposed by Li et al. and we tried to use the difference of the current value and a fixed value. In our trials these thresholds have generated erroneous segmentation in several volumes. Therefore, we decided that the use of one threshold for the entire volume is not as efficient as calculating thresholds for each slice individually. The range of the intensity values in the ROI is still very large. By using a threshold for each image, we can define a more precise threshold for different parts of the volume. The idea on how to calculate the threshold came from the K-means clustering algorithm, where the clustering process is defined as follows:

$$
J=\sum_{j=1}^{K} \sum_{n \in S_{j}}\left|x_{n}-\mu_{j}\right|^{2}
$$

The algorithm clusters the data points into $K$ disjoint classes $S_{j}$ each containing $n_{j}$ data points, where $x_{n}$ is an observation and $\mu_{j}$ is the geometric centroid of the data points in cluster $S_{j}$. Each cluster was initialised with a predefined value. To speed up the process, the classes are calculated from the grey values of the histogram. This means that the algorithm does not need to run over the entire image which would be $512 \times 512$. Instead the classes can be calculated from an array of the size of $1 \times 126$. For each image, the intensity range is partitioned into eight classes which allows putting more weight on the intensity range of the ROI. The eight classes are presented in an array which is used as threshold in the ROI segmentation. During the extraction procedure, the class to which the current voxel will be assigned to, will define the voxel as brain or non-brain tissue.

Region Growing. A region growing algorithm is used to extract the brain tissue. This algorithm considers two thresholds as a stopping condition. The first threshold is used for the identification of the outer boundaries between ROI and CSF in which case we applied the final binary mask. As soon as the algorithm hits a boundary pixel, the region growing stops. The second threshold is used to differentiate the ROI and non-brain tissue within the volume in this case the previous calculated array threshold is applied. To assign the current voxel to a class in that array, the smallest distance between the current voxel and the class centroids is used. In a post-processing step, a local edge refinement 
has been applied which uses the gradient magnitude to refine the outer border pixel. A few automated segmented samples are shown in Figure 4 (top row).

\section{Evaluation}

\subsection{Data Aquisition}

T2 brain volume MRIs (TR: 2660; TE: 142.7; DFOV: $16 \times 16 \mathrm{~cm}$ ) of premature infants have been imaged at full term equivalent in the Children's University Hospital, Dublin, Ireland. Each slice has a thickness of $1 \mathrm{~mm}$ and a dimension of $512 \times 512$ pixels. Our database consists of MRI volumes from five patients. The data of Patient 1 includes 170 images, Patient 2 has 178 images, Patient 3 consists of 186 images, Patient 4 consists of 172 images and the fifth patient includes 88 images. The dataset is composed of a total of 794 images. The first four patients have a slice spacing of $0.5 \mathrm{~mm}$ and the fifth patient has a slice spacing of $1 \mathrm{~mm}$. In order to perform a comprehensive quantitative evaluation of the proposed HSS, the entire database was manually segmented. The manually segmented data has been marked in conjunction with a clinical expert from the Children's University Hospital, Dublin, Ireland.

\subsection{Visual Examination}

Figure 4 (top row) shows automated segmented images sampled from one patient volume. A visual examination of our results indicates the accuracy of the
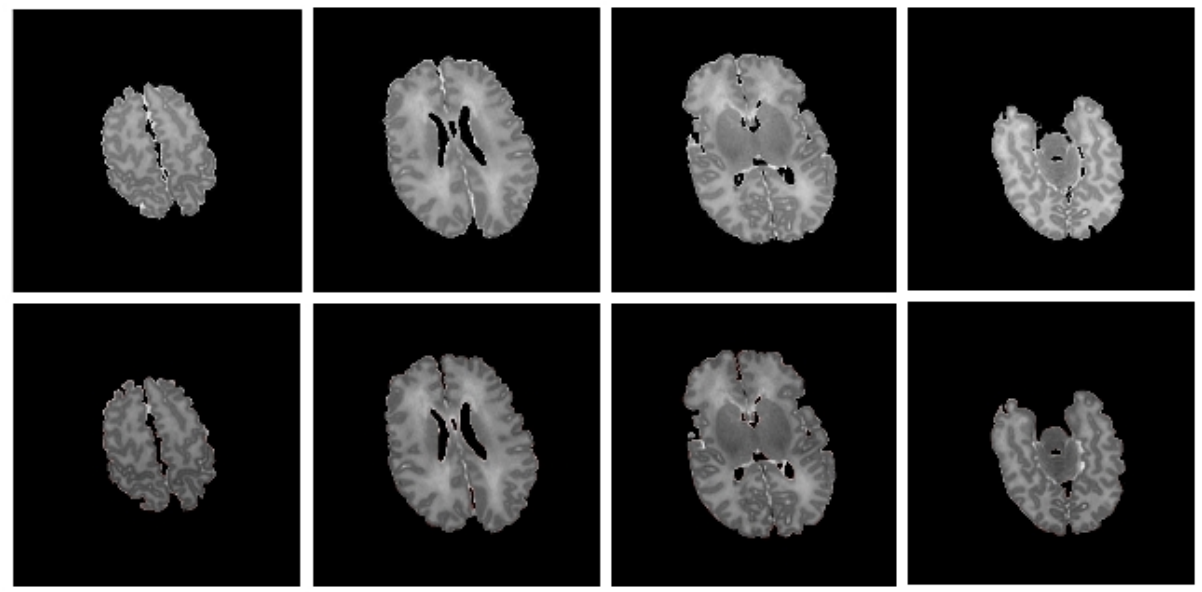

Fig. 4. Top row: result images of the automated skull-stripping segmentation; bottom row: images manually annotated by a clinical expert. The intensity difference between the top and bottom row images is caused by an automatic intensity adjustment in the display of the top row images by Matlab. 
proposed HSS and in order to emphasis this, we have performed a quantitative evaluation when our method is compared against the manually segmented data. During the post-processing step, only the outer boundaries of the ROI were refined but not inside the ROI. Consequently, on some images small parts of CSF can remain. To get a clear opinion on the efficiency of the proposed segmentation method, the results of HSS were compared against the manually segmented data and against four state of the art BEA tools. Each tool, BrainSuite [21], FSL [5] 24], SPM8 [25] and MRIcroN [17], are freely available on the internet, and use one of the three well-established BEA. BrainSuite has embedded BSE and allows the application in a stepwise manner so that the parameters can be adjusted for each step. The only parameter we need to optimize is the size of the structuring element employed in the erosion algorithm in the final step. BET is embedded in two software tools we used. MRIcroN applies BET (version 1) and FSL applies BET2 (version 2). The best results of both tools were obtained using their default values with a fractional intensity threshold of 0.5. Applying SPM8, the best results were provided by adjusting the bias regularisation to a value of 0.1 and using the native space for the generation of GM, WM and CSF.

\subsection{Similarity Metrics and Numerical Evaluation}

1. The first set of tests is done by using the Dice Similarity Metric (DSM) which describes the amount of overlap voxels between the manual segmented data and the automated segmented data. The mathematical formula to calculate the metric is described as follow: $D S M=\frac{2\left|M_{1} \cap M_{2}\right|}{\left|M_{1}\right|+\left|M_{2}\right|}$, where M1 is the automated segmented volume and M2 is the manual segmented volume. The dice similarity metric is a very popular comparison metric used for evaluations in many MRI segmentation approaches [1] 3] [15] [18] 22.

2. The Jaccard metric (JS) measures the similarity between two volumes and has been used as a comparison in previous brain segmentation techniques [16] 18] [19] 22. This is done by dividing the size of the intersection and the size of the union of the two datasets. The mathematical formula to calculate the Jaccard similarity is described as follow: $J S=\frac{\left|M_{1} \cap M_{2}\right|}{\left|M_{1}\right| \cup\left|M_{2}\right|}$

3. Over-segmentation can be calculated using the false positive. This formula calculates the percentage of the amount of voxels which remains in the volume as a part of the ROI but do not belong to the ROI. The false positive was calculated as follow: $F P=\frac{\left|M_{1}-M_{2}\right|}{\left|M_{2}\right|}$

4. Under-segmentation can be calculated using the false negative. This formula calculates the percentage of the amount of voxels which have been removed from the ROI but would belong to the ROI. The false negative was calculated as follow: $F N=\frac{\left|M_{2}-M_{1}\right|}{\left|M_{2}\right|}$

All the comparison results are shown in Table 1 . 
Table 1. Quantitative performance evaluation when the proposed skull-stripping method (HSS) is compared against four state of the art implementations. Best results are highlighted in bold.

\begin{tabular}{lrrrr}
\hline Methodology & DSM & JS & FP(\%) & FN(\%) \\
\hline Patient 1 HSS & $\mathbf{0 . 9 5 8 6}$ & $\mathbf{0 . 9 2 4 9}$ & $\mathbf{4 . 3 4 3 5}$ & 3.9532 \\
FSL & 0.8800 & 0.7858 & 26.8620 & $\mathbf{0 . 3 1 7 1}$ \\
BrainSuite & 0.9065 & 0.8290 & 18.2950 & 1.9336 \\
MRIcroN & 0.7840 & 0.6448 & 38.3500 & 10.7870 \\
SPM8 & 0.9076 & 0.8308 & 16.1460 & 3.5014 \\
\hline Patient 2 HSS & $\mathbf{0 . 9 4 6 2}$ & $\mathbf{0 . 8 9 7 9}$ & 7.0101 & 3.9170 \\
FSL & 0.8793 & 0.7846 & 26.5170 & $\mathbf{0 . 7 4 1 8}$ \\
BrainSuite & 0.9029 & 0.8229 & $\mathbf{1 . 0 5 6 3}$ & 16.8360 \\
MRIcroN & 0.8547 & 0.7463 & 30.7940 & 2.3911 \\
SPM8 & 0.9187 & 0.8496 & 12.5900 & 4.3385 \\
\hline Patient 3 HSS & $\mathbf{0 . 9 6 0 7}$ & $\mathbf{0 . 9 2 4 5}$ & $\mathbf{3 . 7 1 7 8}$ & 4.1182 \\
FSL & 0.8838 & 0.7918 & 26.2120 & $\mathbf{0 . 1 6 0 5}$ \\
BrainSuite & 0.7437 & 0.5919 & 24.6060 & 26.2380 \\
MRIcroN & 0.7097 & 0.5501 & 45.0090 & 20.2370 \\
SPM8 & 0.8386 & 0.7220 & 27.651 & 7.8324 \\
\hline Patient 4 HSS & $\mathbf{0 . 9 5 8 6}$ & $\mathbf{0 . 9 2 0 5}$ & 4.5710 & 3.7439 \\
FSL & 0.8586 & 0.7522 & 32.9050 & $\mathbf{0 . 0 2 7 5}$ \\
BrainSuite & 0.9044 & 0.8255 & $\mathbf{0 . 3 3 9 3}$ & 17.1720 \\
MRIcroN & 0.8144 & 0.6869 & 38.3750 & 49.4460 \\
SPM8 & 0.8733 & 0.7751 & 27.8950 & 0.8640 \\
\hline Patient 5 HSS & $\mathbf{0 . 9 4 7 5}$ & $\mathbf{0 . 9 0 0 4}$ & $\mathbf{3 . 6 0 5 3}$ & 6.7189 \\
FSL & 0.8955 & 0.8107 & 22.4920 & $\mathbf{0 . 6 9 1 3}$ \\
BrainSuite & 0.9262 & 0.8626 & 12.6160 & 2.8591 \\
MRIcroN & 0.8714 & 0.7721 & 25.2660 & 3.2830 \\
SPM8 & 0.8529 & 0.7436 & 15.5720 & 14.0590 \\
\hline & & & &
\end{tabular}

\section{Discussion}

The quantitative results displayed in Table 1indicate that HSS returns accurate results when applied to skull stripping on brain MRI data of premature infants. Table 2 reveals the accuracy of each analysed technique in comparison with the other brain extraction methods by observing the average results of each test. HSS provides the overall best results in the dice similarity, Jaccard similarity and false positive results. The average similarity values are: $95 \%$ for the Dice calculation and $91 \%$ for the Jaccard calculation. There is an average of misclassified voxels of less than $5 \%$ which is a satisfactory result.

Every approach has its strong and weak points which is reflected in the results values in Table 1 and Table 2. HSS has two main weak points which have to be addressed in future work. First, due to the partial volume effect, the boundaries between the lacrimal glands (tear glands) and the ROI are not always visible on the MRI of premature infant. As a consequence, the region growing algorithm will continue to grow in the region instead of excluding it. A second weak point 
Table 2. Average values over the entire database of the results from each comparison test

\begin{tabular}{lrrrr} 
Methodology & DSM & JS & FP(\%) & FN(\%) \\
\hline HSS & $\mathbf{0 . 9 5 4 3}$ & $\mathbf{0 . 9 1 2 7}$ & $\mathbf{4 . 6 4 9 5}$ & 4.4902 \\
\hline FSL & 0.8794 & 0.7850 & 26.9976 & $\mathbf{0 . 3 8 7 6}$ \\
\hline BrainSuite & 0.8767 & 0.7863 & 11.3825 & 13.0077 \\
\hline MRIcroN & 0.8068 & 0.6800 & 35.5588 & 17.2288 \\
\hline SPM8 & 0.8782 & 0.7842 & 19.9708 & 6.1191 \\
\hline
\end{tabular}

consists in the presence of CSF boundaries inside the brain volume. During the pre-processing step only outer boundaries have been refined and for that reason small parts of fluid can remain inside the ROI.

The comparison evaluation revealed that FSL returned the smallest percentage in false negatives but a higher percentage in false positives. This occurs because FSL leaves the CSF in the image and therefore it is less likely to have removed too much of the brain tissue. On the other hand, the higher rate of false positives is caused by the remaining CSF. FSL removes the skull and the fat and only in a few places small parts of the skull can be observed. This technique seems to be a good solution for the removal of skull and fat. In brain MRIs of premature children at the age of a few weeks, the brain structure has not been fully developed. The challenge of early brain development is mainly caused by the fact that the infant brain contains less white matter myelin then the adult brain which results in less defined edges between different regions. Looking at the results generated by BrainSuite and FSL, we observed that they are simular. The major differences can be found in the over segmentation and under segmentation. BrainSuite has average error rates of $11 \%$ for $\mathrm{FP}$ and $13 \%$ for $\mathrm{FN}$ and this might be caused by the edge detection that BSE is based on and the partial volume effect that prevents the edge detection to find the correct boundaries. The problem of PVE has been solved in HSS by using morphological operators to reduce the PVE and by combining the edge detection with morphological operators to enclose the main edges between fluid and brain tissue. MRIcroN uses the first version of BET technique. Same as FSL, MRIcroN does not take the fluid inside the brain volume into consideration. For example, when applying MRIcroN, within an image, on one half CSF and sometimes skull and fat tissue remains and on the other half the CSF is removed but often some of the brain tissue is removed as well. This leads to high error rates. The differences on the results compared to FSL show us that the second version of BET comprise significant improvement. SPM8 is the only technique of these four which was not developed for skull stripping in the first place. The result is presented in three different volumes where each volume represents a different region such as GM, WM or CSF. The similarity and comparison results were calculated by combining the GM and WM images. SPM8 results lie within the same accuracy of FSL and BrainSuite. It has not been stated but in our opinion, SPM8 has been developed to be used on clear structured adult brain MRI and not on children brain MRI with the age of under two years. 


\section{Conclusion}

The purpose of this paper is to introduce an automatic algorithm for the brain extraction from infant MRI data. The developed algorithm is based on a hybrid approach that embeds a suite of image processing tools that include a reduction of artefacts, generation of a binary mask and the application of a region growing for the extraction of the main brain region. One of the advantages of the proposed approach consists in the reduction of PVE, and the numerical results indicate higher performance of the proposed algorithm when compared to state of the art implementations.

Acknowledgment. This study was funded by the Children's University Hospital, Dublin, Ireland. We wish to thank our colleagues for providing us with the medical insight and for expert visual assessment of MRI scans.

\section{References}

1. Babalola, K.O., Patenaude, B., Aljabar, P., Schnabel, J., Kennedy, D., Crum, W., Smith, S., Cootes, T., Jenkinson, M., Rueckert, D.: An evaluation of four automatic methods of segmenting the subcortical structures in the brain. NeuroImage 47, 1435-1447 (2009)

2. Balan, A.G.R., Traina, A.J.M., Ribeiro, M.X., Marques, P.M.A., Traina Jr., C.: Head: The Human Encephalon Automatic Delimiter. In: CBMS 2007: Proceedings of the Twentieth IEEE International Symposium on Computer-Based Medical Systems, pp. 171-176. IEEE Computer Society Press, Washington, DC, USA (2007)

3. Boesen, K., Rehm, K., Shaper, K., Stoltzner, S., Lueders, E., Rottenberg, D.: Quantitative comparison of four brain extraction algorithms. NeuroImage 22, 12551261 (2004)

4. Chiverton, J., Wells, K., Lewis, E., Chen, C., Podda, B., Johnson, D.: Statistical morphological skull stripping of adult and infant MRI data. Computers in Biology and Medicine 37, 342-357 (2007)

5. Crum, W.R., Rueckert, D., Jenkinson, M., Kennedy, D., Smith, S.M.: A framework for detailed objective comparison of non-rigid registration algorithms in neuroimaging. In: Barillot, C., Haynor, D.R., Hellier, P. (eds.) MICCAI 2004. LNCS, vol. 3216, pp. 679-686. Springer, Heidelberg (2004)

6. Friston, K.J., Penny, W.: Posterior probability maps and SPMs. NeuroImage 19, 1240-1249 (2003)

7. Hahn, H.K., Peitgen, H.-O.: The skull stripping problem in MRI solved by a single 3D watershed transform. In: Delp, S.L., DiGoia, A.M., Jaramaz, B. (eds.) MICCAI 2000. LNCS, vol. 1935, pp. 134-143. Springer, Heidelberg (2000)

8. Harnsberger, H.R., Osborn, A.G., Ross, J., Macdonald, A.: Diagnostic and Surgical Imaging Anatomy: Brain, Head and Neck, Spine. Amirsys Inc. (2006)

9. Kobashi, S., Fujimoto, Y., Ogawa, M., Ando, K., Ishikura, R., Kondo, K., Hirota, S., Hata, Y.: Fuzzy-ASM Based Automated Skull Stripping Method from Infantile Brain MR Images. In: IEEE International Conference on Granular Computing, pp. 632-635 (2007)

10. Li, X.: CI, L., Wang, R., Li, J.: A Region Growing Method Based on Fuzzy Connectedness. In: ICALIP, pp. 993-997 (2008) 
11. Marr, D., Hildreth, E.: Theory of edge detection. Proceedings of Royal Society of London 207(B), 187-217 (1980)

12. Mathur, A.M., Neil, J.J., Inder, T.E.: Understanding Brain Injury and Neurodevelopment Disabilities in the Premature Infant: The Evolving Role of Advanced Magnetic Resonance Imagine. Seminar in Perinatology 34, 57-66 (2010)

13. Perona, P., Malik, J.: Scale-Spacing and Edge Detection Using Anisotropic Diffusion. IEEE Transactions on Pattern Analysis and Machine Intelligence 12(7), 629-639 (1990)

14. Pohle, R., Toennies, K.D.: Segmentation of medical images using adaptive region growing. Proceedings of SPIE 4322, 1337-1346 (2001)

15. Prastawa, M., Gilmore, J.H., Lin, W., Gerig, G.: Automatic segmentation of MR image of the developing newborn brain. Medical Image Analysis 9, 457-466 (2005)

16. Rehm, K., Schaper, K., Anderson, J., Woods, R.: Putting our heads together: a consensus approach to brain/non-brian segmentation in T1-weighted MR volumes. NeuroImage 22, 1262-1270 (2004)

17. Rorden, C., Brett, M.: Stereotaxic display of brain lessions. Behavioural Neurology $12,191-200(2000)$

18. Sadananthan, S.A., Zheng, W., Chee, M.W., Zagorodnov, V.: Skull stripping using graph cuts. NeuroImage 49, 225-239 (2010)

19. Segonne, F., Dale, A.M., Busa, E., Glessner, M., Salat, D., Hahn, H.K., Fischl, B.: A hybrid approach to the skull stripping problem in MRI. NeuroImage 22, 1060-1075 (2004)

20. Shanthi, K., Sasi Kumar, M.: Skull stripping and automatic segmentation of brain MRI using seed growth and threshold techniques. In: International Conference on Intelligent and Advanced Systems, ICIAS 2007, November 25-28, pp. 422-426. IEEE Computer Society, Los Alamitos (2007)

21. Shattuck, D.W., Leathy, R.M.: BrainSuite: An automated cortical surface identification tool. Medical Image Analysis 6, 129-142 (2002)

22. Shattuck, D.W., Sandor-Leathy, S.R., Shaper, K.A., Rottenberg, D.A., Leathy, R.M.: Magnetic Resonance Image Tissue Classification Using a Partial Volume Model. NeuroImage 13, 856-876 (2001)

23. Smith, S.M.: Fast robust automated brain extraction. Human Brain Mapping 17, 143-155 (2002)

24. Smith, S., Jenkinson, M., Woolrich, M., Beckmann, C., Behrens, T., JohansenBerg, H., Bannister, P., Luca, M.D., Drobnjak, I., Flitney, D., Niazy, R., Saunders, J., Vickers, J., Zhang, Y., Stefano, N.D., Brady, J., Matthews, P.: Advances in functional and structural MR image analysis and implementation as FSL. NeuroImage 23(S1), 208-219 (2004)

25. SPM8: This software is available at the web address, http://www.fil.ion.ucl.ac.uk/spm/

26. Tzaroushi, L.C., Astrakas, L.G., Zikou, A., Xydis, V., Kosta, P., Andronikou, S., Argyropoulou, M.I.: Preventricular leukomalacia in preterm children: assessment of grey and white matter and cerebrospinal fluid changes by MRI. Pediatric Radiology 39, 1327-1332 (2009)

27. Weickert, J.: Coherence-Enhancing Diffusion Filtering. Internation Journal of Computer Vision 31(2/3), 111-127 (1999)

28. Zhao, W., Xie, M., Gao, J., Li, T.: A Modified Skull-Stripping Method Based on Morphological Processing. In: ICCMS 2010: Second International Conference on Computer Modeling and Simulation, vol. 1, pp. 159-163 (2010) 